\title{
Eating Difficulties in Newly-Onset Stroke Patients: A Qualitative Inquiry of Nurses' Perceptions of the Situation
}

\author{
Wai-see Mavis LAI', Lisa Pau Le LOW' ${ }^{*}$ and Ka Yin Kayla WONG \\ ${ }^{1}$ Prince of Wales Hospital, Hong Kong \\ ${ }^{2}$ School of Health Sciences, Caritas Institute of Higher Education, Hong Kong
}

*Corresponding author: Lisa Pau Le LOW, PhD, MPhil, BN, RHV, RN, Associate Professor, School of Health Sciences, Caritas Institute of Higher Education, 18, Chui Ling Road, Tseung Kwan O, New Territories, Hong Kong, Tel: (+852)-37024232, E-mail: lisalow@cihe.edu.hk

\begin{abstract}
Eating difficulties are one of the challenges experienced by stroke survivors. It is necessary to examine eating difficulties from a broader perspective and not only focus on swallowing. As nurses play a vital role in ensuring good nutrition, greater attention should be given to explore this phenomenon. This paper reports a study to explore nurses' perceptions of caring for newly-onset acute stroke patients with eating difficulties. Twelve registered nurses from two medical wards of an acute hospital in Hong Kong were invited to participate in an interview. Using latent content analysis, five categories resulted to describe the responses nurses took to take care of newly-onset stroke patients with eating difficulties during the acute phase of their illness. The categories 'recognizing patients with eating difficulties' and 'determining swallowing competence as a perquisite to eating' were described by nurses as being the initial responses they took to determine the patient's ability to eat safely. This was followed by the category 'dealing with patients emotions when eating' and 'taking risks and negotiating food choices'. Ultimately, the last category was to prepare family members and the patients to overcome the eating difficulties to enable the patient to be safely discharged home. Nurses should give equal attention to recognizing the cues and mixed emotions of patients confronted with eating difficulties as well as swallowing difficulties. Given that there is a reliance on nurses to help feed the patients, investing time to negotiate safe food choices with the patients and their family members is also needed.
\end{abstract}

\section{Keywords}

Eating difficulties, Nurses, Stroke patients, Qualitative research

\section{Introduction}

Stroke is a sudden and long-term disease. Stroke survivors are known to suffer from various degrees of permanent disability and impairments. Owing to the significant improvement in life expectancy, more stroke survivors are left to survive with certain degrees of disabilities. According to Westergren and colleagues [1], eating difficulties such as chewing and swallowing are one of the challenges stroke survivors may experience. Eating difficulty is defined as 'difficulties that, alone or in combination, negatively interfere with the preparation and intake of served food and or beverages' (p.258). Eating therefore involves not only swallowing but also feeding. The person has to recognize the food, bring the food into the mouth, and chew it before it can be swallowed [2]. Indeed, it is necessary to examine eating difficulties from a broader perspective and not only focus on swallowing. Other issues that relate to the eating difficulties of stroke patients include poor sitting positions, inability to manipulate the food between the plate and mouth, poor handling of cutlery, and communication problem that can hinder their meal preferences from being known [3]. As nurses play a vital role in ensuring good nutrition, examining nurses' perceptions of caring for stroke patients with eating difficulties should receive greater attention.

Previous studies have found that the eating experiences of stroke survivors to be rather negative over time $[4,5]$. Among 30 acute stroke patients admitted to 
an internal medicine unit and a neurological unit in Sweden [4], the findings demonstrated that stroke patients expressed fear of choking, discomfort in the mouth and throat, thirst related to difficulty drinking from a glass, and feeling shameful about being dependent. The patients emphasized that eating was no longer enjoyable or as pleasurable as before. They perceived eating to be embarrassing owing to a lack of oral control and a need to be in a rush to finish their meals. They felt that healthcare personnel were not aware or did not understand their feelings.

Medin and colleagues [5,6] supported that eating problems can persist three months after the stroke, with no significant changes to the psychological well-being [7]. For those who had survived the stroke after six months, Perry and McLaren [8] continued to reveal persistent difficulties associated with eating. Such difficulties included cutting and eating food due to restricted limb movements, chewing difficulties and being unaware of the food temperature. The patient's psychological reactions also varied from bewilderment through to dismay and despair. Indeed, the patients desired to either return to their premorbid state or to accept their condition by using adaptive approaches such as choosing modified texture diets. Furthermore, even up to two years after the first stroke, patients described feeling abandoned when managing their eating problems and perceived that nursing staff lacked the knowledge to help them with eating difficulties [9]. Consequently, patients did not receive adequate training, practical advice and support with their meals at the hospital. It would seem that striving for independence was evident among patients with eating difficulties. This claim is supported by a Swedish study that investigated cultural values and table manners of older patients who expressed strong needs to manage in a normal way and to be self-sufficient in their table manners [10]. Although the meal procedures were simplified, patients felt shameful and were actually reluctant to disclose their personal needs to the nurses as they were guided by the norm to be 'contented'. Additionally, another study described eating difficulties and dysphagia, types of nursing intervention and the development of complication of $30 \mathrm{pa}-$ tients over a three-month period after the stroke [11]. The level of alertness and energy were found to be pertinent to the patient's ability to eat and swallow, and the development of complications over time. Therefore, staff caring for these patients would need to target at ways that addressed eating difficulties such as manipulating food on the plate, transporting it to the mouth, aberrant eating speed, sitting position and opening or closing the mouth were encountered [12].

Regarding attitudes and knowledge about nutrition and food service in an acute hospital, nurses believed they played an important role in assisting the patients to eat. However, several nurses saw feeding as an un- enjoyable and a low priority task and were under pressure to complete other routines [13]. Similarly, nurses working in an acute care hospital were found to be busy with activities such as documentation and medication during mealtimes and could not provide timely help for patients with eating difficulties [14]. From a nursing and health promoting perspective, it is important to invest attention to understanding patient's inability to chew the food and to explore safe eating solutions to deal with malnutrition and aspiration. According to Abe and colleagues [15], the importance of chewing which prepares the food for swallowing and, therefore, the absence of teeth or the poorly fitted dentures can increase the risk of aspiration was highlighted. Indeed, poor intake of food may result from difficulties in swallowing. In fact, many stroke patients are unable to eat or drink independently because of the inability to swallow, reduced level of consciousness, low mood, weakness of limbs as well as other physical and functional problems [16]. The literature suggests that stroke survivors had eating difficulties and perceived nurses to be rather unhelpful and not knowledgeable in helping them to combat these difficulties. Therefore, this study specifically explored the nursing care of newly-diagnosed stroke patients with eating difficulties.

\section{Methods}

\section{Design}

A descriptive qualitative design was used to explore nurses' perceptions of eating related issues encountered among newly-onset stroke patients.

\section{Settings and participants}

Two mixed gender medical wards of an acute hospital in Hong Kong were selected. Each ward comprised 32 beds in which six beds from each ward were designated to the acute stroke unit. The average length of stay of stroke patients was between four to five days. All new cases of stroke were diagnosed within seven days and were admitted to the stroke unit. When there were no vacant beds, these patients would be in the general medical wards until beds in the stroke unit became available. The two wards provided the same care and used similar protocols for managing stroke patients, including the stroke care pathway, swallowing management team protocol and swallowing screening form [17]. Convenience sampling was used to recruit 12 fulltime registered nurses working in the medical wards with at least two years of working experience in the unit. Enrolled nurses were excluded as they were not assigned to be in-charge of stroke patients in the stroke unit. Ward managers and Department Operation Manager were also excluded as bedside care was not their main scope of work. The recruitment process involved making an announcement about the purpose, methodology and inclusion criteria of the study during the shift handover for three consecutive days. An information 


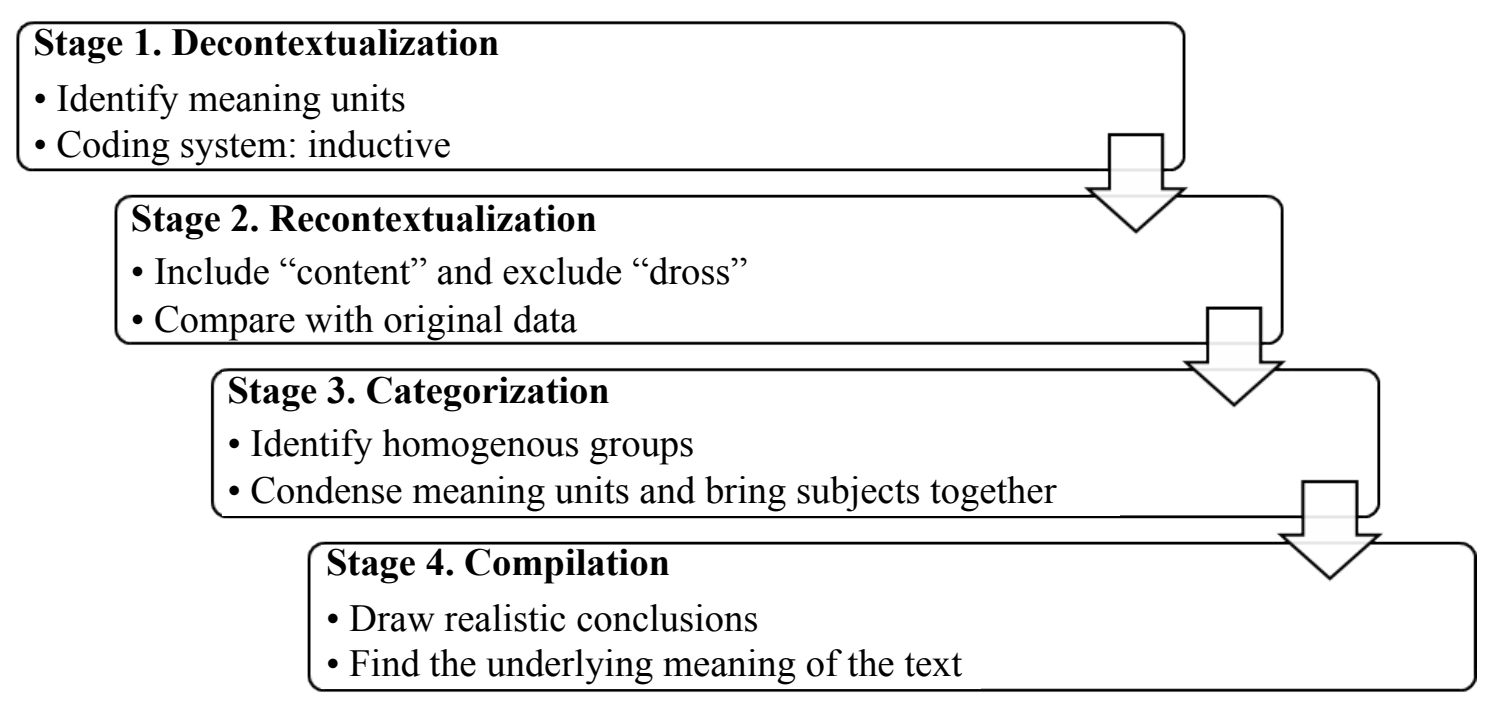

Figure 1: Overview of the content analysis process.

sheet was distributed during the briefing session and participants voluntarily signed up if they were interested to participate.

\section{Ethical considerations}

Ethical approval was obtained from the research ethics committee of the university and the hospital concerned. Participants were informed of the rights to refuse to answer any questions and to terminate the interview at any time. Consent forms were signed before conducting the interviews.

\section{Data collection}

Once ethical approval was obtained, a demographic data sheet collected information about the rank, gender, years of nursing experience, education level, and training in stroke care from the participants. The interview guide was developed from the literature review and commented by an expert nurse who had worked in stroke care for the past 12 years. The broad questions used in the interviews were as follows:

- What eating difficulties do you think stroke patients are experiencing?

- What do you see as the feelings that stroke patients with eating difficulties will have?

- What are you currently doing in caring for stroke patients with eating difficulties?

- How do you feel when you feed stroke patients with eating difficulties?

- What are the barriers/difficulties you have experienced when caring for these patients?

The first author, who was undertaking a postgraduate degree, conducted the interviews and had prior understanding as a registered nurse on the needs and care of stroke patients. The date and time of the 45 minutes interview were negotiated with each participant. Many of them chose to be interviewed during their off-duty hours. Field notes were recorded to document what the researcher had seen, thoughts they had, and the participants' nonverbal cues during the interviews.

\section{Data analysis}

Simultaneous data collection and analysis were conducted using latent content analysis [18]. Each audio-taped interview was transcribed verbatim. The accuracy of each transcript was checked by rereading them several times before assigning codes to the meaningful segments in order to make sense of the data. According to Patton (chapter 8, p.431-514) [19], categories were developed based on two criteria: (1) The extent to which the data that belong in a certain category dovetail in a meaningful way; and (2) The extent to which the differences among categories were clear. Figure 1 shows an overview of the content analysis process $[18,19]$. This essentially involved sifting the data and putting the codes into a comprehensive whole. Different phrases, relationships and patterns in the data were identified and compared for similarities. The final emergent categories were compared and refined with the new incoming data from the interviews. Data saturation was achieved in this study when repetitive and duplicated information were obtained from the participants. A framework comprising the responses from the nurses who took care of newly-onset acute stroke patients with eating difficulties was created.

\section{Rigor}

We took the following measures to ensure rigor, particularly to ensure the credibility of the data, which refers to the truthfulness of the data in reflecting the participants' experiences of the phenomenon under study. Measures included inviting a nurse with expertise in stroke care to review and validate the relevance and appropriateness of the interview guide. All interviews were undertaken in Cantonese and translated directly to English verbatim for analysis. In ensuring translation accuracy, a bilingual speaking nurse with a master de- 
Table 1: Demographic data of nurses.

\begin{tabular}{|l|l|l|l|l|l|l|l|}
\hline No. & Rank & Gender & Post-RN (Years) & Post-EN (Years) & Years in ward & Education & Stroke training \\
\hline N1 & RN & F & 6 & NA & 4 & Bachelor & Nil \\
\hline N2 & RN & F & 1 & 10 & 11 & Bachelor & Nil \\
\hline N3 & RN & F & 8 & NA & 4 & Bachelor & Nil \\
\hline N4 & APN & F & 19 & NA & 10 & Baster & Nil \\
\hline N5 & RN & F & 4 & NA & 2 & Master & In-house training \\
\hline N6 & APN & F & 19 & NA & 4 & Bachelor & Cert. in neuroscience \\
\hline N7 & RN & F & 11 & NA & 10 & Master & Cert. in neuroscience \\
\hline N8 & APN & M & 13 & NA & 10 & Bachelor & Nil \\
\hline N9 & RN & F & 10 & NA & 10 & Bachelor & Nil \\
\hline N10 & RN & F & 3 & NA & 2 & Bachelor & Cert. in geriatric nursing \\
\hline N11 & RN & M & 10 & 6.5 & 6 & Bachelor & Nil \\
\hline N12 & RN & F & 4.5 & 11 &
\end{tabular}

Note: N: Nurse; RN: Registered Nurse; EN: Enrolled Nurse; APN: Advanced Practice Nurse.

gree in health science was invited to translate the first interview with the first author. A high level of equivalence between the two transcripts was found. Keeping accurate audiotaped interviews and field notes were done to ensure the consistency of the collected data.

\section{Findings}

The demographic data of 12 registered nurses is shown in Table 1.

The analysis resulted in five categories that characterized the responses from nurses when they took care of newly-onset stroke patients with eating difficulties during the acute phase of illness. 'Recognizing patients with eating difficulties' and 'determining their swallowing competence' were the initial responses nurses described as crucial to deciding the patient's ability to eat safely. As patients realized their eating patterns had altered, 'dealing with the patients emotions on eating' and 'taking risks and negotiating food choices' triggered nurses to respond by providing support and emphasizing on the continual need to take precautions when feeding the patients. The last category was to prepare family members and patients to overcome the eating difficulties to enable the patient to be safely discharged.

\section{Recognizing patients with eating difficulties}

One of the initial responses of nurses in caring for newly-diagnosed stroke patients was to recognize the types of eating difficulties that stroke patients could face. 'Inability to swallow' was identified as one type type of swallowing functions affecting these patients after the stroke. These patients could not swallow well, choked easily and food would drool whilst eating. The 'inability to resume usual diet' was the second type of eating difficulties nurses perceived stroke patients to have. This was when the patients were unable to tolerate the food consistency they had before the admission. A third type of eating difficulty that could arise was the 'inability to eat psychologically'. This was defined as due to the stroke itself which bought on feelings of patients not wanting to eat any more:

"Patients' refusal to eat may be caused by psycho- logical factors like poor self-perception, self-image and depressed mood. These factors can make them unhappy and refuse to accept their condition so they refuse to eat". (N4)

Another type of response related to the 'inability to feed self'. One nurse said:

"It's difficult for them to use only one functional limb to feed (themselves). Even when they've no problems in eating they may be unable to use the cutlery". (N11)

Finally, the 'inability to chew' the food was mentioned particularly when most of the patients on the wards were old and did not have intact teeth.

\section{Determining swallowing competence as a prereq- uisite to eating}

The second category was the need of nurses to carefully determine the swallowing competence of the patients before they even begun to eat. As a gold standard, swallowing competence was initially assessed using the bedside water-swallow test. Nurses would strictly adhere to this protocol when assessing the patients' swallowing competence and were knowledgeable about the signs of swallowing difficulties (that is, no choking, coughing and shortness of breath). When nurses could not determine whether patients had choked because they were in a hurry to drink water or they had swallowing problems, patients would be kept nilby-mouth. Some nurses found that although the bedside water-swallow test allowed them to have a rapid and simple initial screening tool, they desired to receive more training about the assessment to reduce the risk of choking, especially during times when the speech therapist was not present to give advice.

"Swallowing test is reliable enough for initial screening. It can be regarded as a simple screening before the speech therapist comes. However, we can observe the patients more thoroughly if we know more about the assessment during the holidays. This can reduce the risk of choking". (N6)

For patients who had passed the test, nurses found 
that they were still hesitant about allowing them to resume the usual diet while others resorted to modified diet with varied consistency instead, until these patients were reassessed by the speech therapist to confirm the nurses' judgment. For those failing the test, no food was allowed until more assessment was performed by the speech therapist.

\section{Dealing with patients emotions when eating}

Once the patients eating difficulties had been identified, with decisions made to either resume or withhold diet, patients went through various emotions as they dealt with their eating issues. Although nurses recognized these mixed emotions, time constraint had prevented them from responding to the patients as it took time to deal with their emotions. There was a set of emotions relating to 'not knowing what's happening' to them. Some patients who failed the bedside water-swallow test demonstrated emotions of bewilderment because they were being fasted for the speech therapist's assessment. Some queried why they were given modified diet and were unclear whether they had to continue eating such food in the future:

"They usually worry and are afraid they need to eat this food in the future. They aren't clear about how things will go". (N3)

Nurses also had to deal with patients who were 'denying the situation'. Although some nurses found that patients tolerated with being told to be fasted or ate modified diet, they were nevertheless complaining about the arrangement:

"Some patients ask why they've to eat this pureed diet and prefer not to eat it. They believed they can have normal rice (to eat). She chooses not to eat. I can do nothing (about it)". (N1)

As time went by, nurses reported that the patients were 'losing interest in their food'. While the nurses acknowledged the poor texture of the pureed food and the tasteless thickened fluid, they needed to be reminded to demonstrate more empathetic and supportive attitude:

"Most patients don't like pureed food because they can't chew the texture. While there's less chance of puree going into the wrong compartment, they loose the joy of eating. Vegetables aren't like vegetables, meat isn't like meat". (N6)

Furthermore, 'feeling dependent on others to eat' was another set of patients' emotions nurses had described:

"They're frustrated because they've become more dependent suddenly. Eating is a very basic skill of human beings. They've to depend on others and they can't swallow the food. They're frightened, disappointed and unhappy". (N12)
Indeed, learning to deal with the patients' range of emotions and the continual need to take precautions to monitor their eating was further emphasized in the next category.

\section{Taking risks and negotiating food choices}

As patients worked through various emotions, some were still reluctant to accept their eating difficulties and resorted to taking risks to try different food choices. While nurses would follow the speech therapist's recommendation to provide modified diets and did not want to take risks, few nurses allowed the patients to experience risks by eating what they liked. For patients who could not accept, water was given to them to try and to learn the truth about their risk of choking. When choking was observed, patients would be more reluctant about drinking fluids again.

Meanwhile some patients were assisted by their family members to take risks. A dilemma presented to the nurses was the risks arising from family members who brought in food the patient liked, but were risky:

"Totally uncooperative patients will ask their family to bring 'siu mei rice' (roasted pork and rice). This is a dilemma for us. We try our best to explain, but if the patients insist on eating those foods, they have to take risk themselves". (N2)

In order to find a balance between patients' safety and preferences, nurses tried to help the patients by negotiating safe food choices. Importantly, there was a proactive attitude to call the speech therapist to review the patient's condition and whether the diet could be upgraded (that is, from pureed to a soft diet). Although nurses would like to be able to negotiate food choices with the patients, they hesitated about who should shoulder the responsibility: "We're not (speech) therapists. We're not professional enough to determine diet consistency". (N10) The dietitians were also consulted to maximize the patients' food choices. When it was not possible to resume normal diets, nurses allowed the patients to experience eating 'normally':

"When I feed them, there're separate portions containing the pureed vegetables, meat and rice. I don't like to mix them together. I feed them the pureed dishes separately, just like how a normal person eats". (N5)

Lastly, taking safety precautions to monitor the patient's feeding occurred when their conditions fluctuated and continuous monitoring was needed; for example, with the high-risk patients with dysphagia. However, insufficient staffing made it difficult for nurses to perform the feeding, and was the norm to delegate to the health care assistants. Although these assistants had passed competency assessment in feeding and would report to the nurses should problems arose, concerns over the high turnover and training issues such as supervising feeding skills and feeding high-risk patients were highlighted. 


\section{Preparing to overcome eating difficulties}

The final category was to prepare patients to overcome eating difficulties in order to be discharged. This was achieved when nurses instilled hope and worked with the family as they prepared the patients to transit from the acute to rehabilitation phase. Instilling hope was described when nurses told the patients that their conditions may improve and their diet had been upgraded:

"They're more at ease when they know there's room for improvement. We can't tell her firmly that she'll have normal food later but she can try. We give her hope so she can be more hopeful". (N3)

Nurses recognized the role of the family in helping stroke patients to go through their eating difficulties and continue their rehabilitation. This essentially meant working with the family and asking the family to support and encourage the patients. Meanwhile, some nurses described stroke patients as being dependent on the family to look after them following discharge. Therefore, it was perceived to be important to provide the family with information in order to help the patients to adapt to their eating difficulties and prevent complications:

"We should teach patients and family members to use the thickener and recognize signs of aspiration. Patients depend on their family to care for them after discharge so it's better if we can educate the family earlier". (N12)

\section{Discussion}

The findings of this study revealed that most nurses primarily focused on swallowing amidst the other eating difficulties identified among newly-diagnosed stroke patients. As mentioned in the introduction regarding the inability to chew the food, Abe and colleagues [16] highlighted that chewing actually prepares the food for swallowing and, therefore, it needs to be noted that the absence of teeth or the poorly fitted dentures can increase the risks of aspiration. Indeed, many stroke patients can encounter the inability to eat or drink independently owing to swallowing difficulties, reduced consciousness, low mood and other physical and functional problems as well [17]. Previous studies also demonstrated that stroke patients perceived various eating difficulties including problems in manipulating food on the plate and manipulating food in the mouth [4], difficulties in transporting food to mouth, sitting in appropriate position to eat, sensing the food, and possessing adequate energy to eat [11]. However, only few nurses in this study addressed stroke patients' difficulties in feeding themselves, and mainly emphasized on the swallowing ability of stroke patients as attributed to the consequences of dysphagia.

Given that the incidence of dysphagia in patients with acute stroke can vary, nurses may regard swallow- ing problems as the most striking and specific eating difficulty that these patients can encounter. In this study, the bedside water-swallow test was used to identify patients at risk of dysphagia and allowed nurses to screen stroke patients before the speech therapist's assessment. Barnard [20] emphasized the implementation of evidence-based clinical guidelines and screening protocols for dysphagia to all stroke patients regardless of the stroke severity in order to improve patient outcomes. Indeed, early nursing interventions that included observing for swallowing difficulties, performing early screening and advocating suitable treatments can help to prevent complications of dysphagia [20]. Therefore, nurses should be more involved in a multidisciplinary team and contribute to the overall management of dysphagia of their patients [20].

While findings of this study highlighted nurses concerns over the patient's inability to resume premorbid diet, only a few of them noticed the psychological impact of the patients' eating ability especially when it is known that post-stroke depression can adversely affect the patients' functional abilities. While Medin and colleagues $[5,6]$ supported that eating problems can persist three months after the stroke with no significant changes to the psychological well-being [15], other studies have revealed that nurses believed that dealing with the patients' emotions was crucial in caring for stroke patients with eating difficulties as opposed to only focusing on the physical care and interacted in a routine and superficial way with limited emotional interaction [21]. Although nurses in this study had empathetic attitudes when addressing the patient's emotions, they perceived that this would require them to invest in a lot of time. Findings of this study also revealed that patients had emotions about not knowing what was happening and denying the situation. As such, patients were often abandoned to deal with their eating difficulties because they lacked support and time from nursing staff. Owing to the sudden and unpredictable onset of stroke, these patients were often unprepared to deal with its impact on their daily life. Consistent with Jacobsson, et al. [4], feeling 'loss of joy in eating' and 'humiliating dependence' in the eating experience of acute stroke patients were also identified in this study. Similarly, Whitening and Humter [22] supported that as eating was largely a social activity, being kept fasted can create emotional impacts and stigma for the sufferers.

Unsurprisingly, nurses in this study faced challenges when stroke patients and their families took risks to try alternative food choices when they should be having modified diets. 'Allowing patients to take risk' seemed to be an approach adopted in this study to let patients get more knowledge about complications. This study found that nurses adopted approaches to negotiate safe food choices with the patients and allowed them to participate in making decisions. They also actively consulted rather than merely waited for the speech 
therapist's reassessment to upgrade the patient's diet. They believed that serving food as 'normally as possible' could enhance food consumption in the patients. However, attention should be given to the presentation and portion size because unrealistically large portion of food can be overwhelming. This study reflected that nurses relied on the health care assistants to feed the patients but were concerned about the implications of safe patient care. However, there were minimal data from the nurses themselves about ways to actually maintain or improve the nutrition of stroke patients. According to Terrado, et al. [2], the family and patients had the right to refuse the recommended diet but they should be properly informed about the potential consequences of their decisions, including malnutrition and aspiration.

Current findings of this study can be supported by the person-centered care approach, which emphasizes that patients should be given the opportunity to take part in their care planning [23]. In rehabilitating patients with dysphagia, education should include the use and effectiveness of feeding strategies and swallowing techniques for both stroke patients and family caregivers. Family meetings can be incorporated to help avoid misunderstanding and resolve conflicts in caring [23]. Therefore, it is crucial to promote awareness of eating difficulties, provide relevant assistance during meals, improve patients' knowledge about appropriate food choices and increase discussion with the patients about their eating difficulties [24].

Indeed, stroke patients in this study who were perceived to be more hopeful by the nurses were more likely to believe they could overcome obstacles and attain meaningful goals. After the acute phase of stroke, the existence and severity of eating difficulties had a significant impact on the patients after discharge. Apart from instilling hope in accordance with the severity of stroke, it is imperative for nurses to help patients to reset or redefine their goals in response to changing circumstances. Previous studies have found that many patients could return home with the help of the caregivers and family members if they had good mobility and swallowing function [25]. The provision of information to the patients, especially the flow of care and treatment plan and the reasons for eating difficulties should be reiterated. Nurses are responsible for questioning the lengthy periods that patients are fasted, and ensuring that other methods of nutrition and hydration support are established [26]. Person-centered care is also important in planning discharge for stroke patients with swallowing problems. Health care staff and nurses should conduct assessment and set goals before and after discharge for stroke patients by prescribing nutritional supplements and maintaining hydration. The provision of refresher training and ongoing regular support on safe swallowing practices, mouth care, food preparation and texture-modified diets should be a priority for all staff engaged in feeding stroke patients [23].
Another important finding from this study was the involvement of the patients' family members. In the Chinese philosophies of Confucianism, family relationship is obligatory and bound by prescribed roles in the formal kinship norms [27]. This study found that nurses should involve the family by giving information that included reinforcing chewing and swallowing strategies, correct positioning, pacing the rate of intake, and creating a relaxed, supportive and pleasant atmosphere during mealtimes. The educational needs of stroke patients and caregivers would include the provision of knowledge on the clinical aspects, prevention, treatment and functional recovery of stroke. However, owing to the heavy workload, it can be difficult for nurses to communicate the patients' needs to the family members. These findings can be supported by the family-centered approach to care, which encourages the collaboration between the patients, family caregivers and health care staff in the planning, delivery and evaluation of care provided. Such an approach can be the basis for expanding the knowledge of staff on the long-term impact of illness and issues on transiting back home. Particularly, the use of family-centered strategies can help nurses provide appropriate and individualized care of stroke patients during the rehabilitation period [28].

\section{Limitations}

As nurses were recruited from one site, hospital practices and protocols might have influenced their perceptions of caring for stroke patients with eating difficulties. The results might be subjected to recall bias as nurses subjectively recalled their experiences. Only registered nurses were recruited and other grades of nurses such as enrolled nurses could be recruited in future study to examine if differences exist in their perceptions. Although the two wards were similar in organizational structure, staffing levels and used the same protocols, different perceptions could have arose as they cared for stroke patients with different gender.

\section{Conclusions}

The analysis resulted in five categories to characterize the responses of nurses who took care of newly-onset stroke patients with eating difficulties. The findings not only identified the knowledge deficit and strategies adopted but also the problems nurses faced while caring for these patients. Nurses in this study primarily focused on swallowing amidst various eating difficulties. This indicated a need for nurses to broaden their assessment to recognize and assess eating difficulties other than impaired swallowing. Additionally, in considering the impacts of emotions on post-stroke recovery of the patients, recognizing the patient's cues and mixed emotions in relation to eating difficulties will be another aspect of nurses' assessment of newly-onset stroke patients. Definitely, regular refresher course, updates and sharing should be planned to enable all staff to participate in the identification of such issues and provide care for this client group. 


\section{References}

1. Westergren A, Karlsson S, Andersson P, Ohlsson O, Hallberg IR (2001) Eating difficulties, need for assisted eating, nutritional status and pressure ulcers in patients admitted for stroke rehabilitation. J Clin Nurs 10: 257-269.

2. Terrado M, Russell C, Bowman JB (2001) Dysphagia: An overview. Med Surg Nurs 12: 233-250.

3. Jacobsson C, Axelsson, Wenngren BI, Norberg A (1996) Eating despite severe difficulties: Assessment of poststroke eating. J Clin Nurs 5: 23-31.

4. Jacobsson C, Axelsson K, Osterlind OP, Norberg A (2000) How people with stroke and healthy older people experience the eating process. J Clin Nurs 9: 255-264.

5. Medin J, Windahl J, von Arbin M, Tham K, Wredling R (2011) Eating difficulties among stroke patients in the acute state: A descriptive, cross-sectional, comparative study. J Clin Nurs 20: 2563-2572.

6. Medin J, Windahl J, von Arbin M, Tham K, Wredling R (2012) Eating difficulties among patients 3 months after stroke in relation to the acute phase. J Adv Nurs 68: 580589.

7. Carod-Artal FJ, Egido JA (2009) Quality of life after stroke: The importance of a good recovery. Cerebrovasc Dis 27: 204-214.

8. Perry L, McLaren S (2003) Eating difficulties after stroke. J Adv Nurs 43: 360-369.

9. Carlsson E, Ehrenberg A, Ehnfors M (2004) Stroke and eating difficulties: Long-term experiences. J Clin Nurs 13 825-834.

10. Sidenvall B, Fjellstrom C, Ek AC (1996) Cultural perspectives of meals expressed by patients in geriatric care. Int $\mathrm{J}$ Nurs Stud 33: 212-222.

11. Westergren A, Ohlsson O, Rahm Hallberg I (2001) Eating difficulties, complications and nursing interventions during a period of three months after stroke. J Adv Nurs 35: 416426.

12. Westergren A, Ohlsson O, Hallberg IR (2002) Eating difficulties in relation to gender, length of stay and discharge to institutional care among patients in stroke rehabilitation. Disabil Rehabil 24: 523-533.

13. Kowanko I, Simon S, Wood J (1999) Nutritional care of the patients: Nurses' knowledge and attitudes in an acute care setting. J Clin Nurs 8: 217-224.
14. Xia C, McCutcheon $H$ (2006) Mealtimes in hospital-who does what? J Clin Nurs 15: 1221-1227.

15. Abe S, Ishihara K, Adachi M, Okuda K (2006) Oral hygiene evaluation for effective oral care in preventing pneumonia in dentate elderly. Arch Gerontol Geriatr 43: 53-64.

16. Rowat A (2011) Malnutrition and dehydration after stroke. Nurs Stand 26: 42-46.

17. Hospital Authority (2016) Appropriateness of care. Strategic service framework for rehabilitation services. Hong Kong, 58-60.

18. Bengtsson M (2016) How to plan and perform a qualitative study using content analysis. NursPlus Open 2: 8-14.

19. Patton M (2002) Qualitative research \& evaluation methods. ( $3^{\text {rd }}$ edn), Sage Publications, California.

20. Barnard SL (2011) Nursing dysphagia screening for acute stroke patients in the emergency department. J Emerg Nurs 37: 64-67.

21. Gordon C, Ellis-Hill C, Ashburn A (2009) The use of conversational analysis: Nurse-patient interaction in communication disabling after stroke. J Adv Nurs 65: 544-553.

22. Whitening $\mathrm{N}$, Hunter $\mathrm{J}$ (2008) Nursing management of patients who are nil by mouth. Nurs Stand 22: 40-45.

23. (2012) Best care for older people everywhere. State of Victoria, Department of Health, Victorian Government, Melbourne.

24. Pedersen PU, Tewes M, Bjerrum M (2012) Implementing nutritional guidelines - the effect of systematic training for nurse nutrition practitioners. Scand J Caring Sci 26: 178185.

25. Maeshima S, Osawa A, Miyazaki Y, Seki Y, Miura C, et al. (2011) Influence of dysphagia on short-term outcome in patients with acute stroke. Am J Phys Med Rehabil 90: 316-320.

26. Coxall K, Dawes E, Forsyth E, Lloyd H (2008) Applying the key principle of nutrition to nursing practice. Nurs Stand 22: 44-48.

27. Sit WH, Wong KS, Clinton M, Li LS (2007) Associated factors of post-stroke depression among Hong Kong Chinese: A longitudinal study. Psychol Health Med 12: 117-125.

28. Creasy KR, Lutz BJ, Young ME, Stacciarini JM (2015) Clinical implications of family-centered care in stroke rehabilitation. Rehabil Nurs 40: 349-359. 